\title{
Desempenho na Leitura. Crianças Portuguesas e Espanholas. Estudo Comparativo, Exploratório, com a Utilização da PROLEC-R
}

\author{
Ana Paula Couceiro Figueira \\ Jennifer Merçon \\ Faculdade de Psicologia e de Ciências da Educação, Universidade de Coimbra \\ Coimbra, Portugal
}

\begin{abstract}
RESUMO
Os pressupostos deste estudo assentam na assunção da importância da leitura enquanto forma de aquisição de conhecimentos, de enriquecimento cultural e social, bem como de prazer e entretenimento, e, igualmente, da constatação da escassez de medidas nacionais fiáveis, válidas e aferidas nacionalmente de avaliação deste processo complexo. O objetivo último é contribuir para a aferição portuguesa da Bateria de Avaliação dos Processos de Leitura para Crianças, a PROLEC-R, para crianças dos 6-12 anos, explorando o desempenho numa amostra de crianças portuguesas, comparando com uma amostra de crianças espanholas do $5^{\circ}$ ano de escolaridade. Verificamos uma acentuada dificuldade dos alunos de ambas as amostras nas provas Estruturas Gramaticais, Compreensão de Textos e Compreensão Oral, não se registando diferenças nem dificuldades acentuadas nas restantes provas. Concluímos pela afirmação da necessidade de mais estudos.
\end{abstract}

Palavras-chave: Leitura; Desempenho na leitura; Comparação intercultural; Estudo exploratório; Adaptação de instrumentos.

\section{ABSTRACT}

Performance in Reading. Portuguese and Spanish Children. Comparative Exploratory Study Using the PROLEC-R This study are based on the assumption of the importance of reading and also the finding of lack of reliable, valid and nationally benchmarked national measures of the assessment of this complex process. This study had two goals: the main goal of the study was to contribute to the Portuguese assessment of a Battery for Children Reading, the PROLEC-R, aimed at children aged 6-12 years. In this exploratory study, the purpose is also to qualitatively compare the performance of Portuguese and Spanish children in the 5th grade of primary school. We can see a marked difficulty for students of both samples in the tests Grammatical Structures, Understanding Texts and Listening, and which does not marked differences in the remaining races. We conclude of the need for more studies.

Keywords: Reading; Reading performance; Intercultural comparison; Exploratory study; Adaptation of instruments.

\section{RESUMEN}

Rendimiento en Lectura. Niños Portugueses y Españoles. Estudio Exploratorio, Comparativo, Utilizando el PROLEC-R

Este estudio se basa en la asunción de la importancia de la lectura y también el hallazgo de la falta de medidas nacionales fiables, válidas de evaluación de este complejo proceso. El objetivo final de este estudio es contribuir a la adaptación para Portugal de la batería PROLEC-R, para los niños 6-12 años, con la exploración de la realización de una muestra de portugués y español de los niños $5^{\circ}$ grado, comparando los resultados. Se observa una marcada dificultad para los estudiantes de ambas muestras en las pruebas de estructuras gramaticales, comprender textos y la comprensión por escucha, más no lo hace diferencias o dificultades notables en las carreras que quedan. Llegamos a la conclusión, dada la naturaleza exploratoria del estudio, la declaración de la necesidad de más estudios.

Palabras clave: Lectura; Rendimiento en la lectura; Comparación intercultural; Estudio exploratorio; Adaptación de instrumentos. 
A importância da leitura é indiscutível. Apesar das divergências de opiniões, ela é uma "forma de prazer, de aquisição de conhecimentos e de enriquecimento cultural, de ampliação das condições de convívio social e de interação" (Soares, 2000, p. 19). Assim, para garantir que qualquer ser humano obtenha sucesso escolar, profissional e social (Borges, 1998; Carvalho \& Pereira, 2008; Sousa, 1999) e que se torne um cidadão autónomo, é imprescindível que domine bem a leitura (Leite \& Bragança, 2010; Paulino, 2009).

No entanto, a leitura não é adquirida de forma espontânea (Silva, 2003). É uma atividade complexa que resulta de diversos processos cognitivos (por ex: a perceção da fala, a consciência fonológica, o conhecimento alfabético, o acesso ao léxico, a velocidade de processamento, o processamento morfológico, o processamento ortográfico, o processamento sintático, o processamento semântico, a memória de trabalho, a atenção, etc.), que o leitor utiliza perante a construção do sentido de um texto (Campos, 2008; Cruz, 2007). Neste sentido, é fundamental estimular e promover a prática da leitura (Borges, Assagra, \& Alda, 2010), reconhecendo o seu valor e compreendendo os mecanismos de aquisição, os seus processos, os componentes e os métodos de ensino, no sentido de se operacionalizar uma forma de atuação mais eficaz e consciente para os seus principiantes (Esteves, 2008).

A complexidade deste processo vem, então, esclarecer a razão por que são realizadas as mais variadas investigações no campo da literacia, ou capacidade de ler e de escrever e capacidade para perceber e interpretar o que é lido (Soares, 1991). Cada vez mais se multiplicam as conferências sobre a promoção da leitura, os relatórios sobre os alunos dos diversos países e as advertências aos diferentes governos para que se adote a promoção da leitura como uma prioridade política (Velasquez, 2007). Resultados alcançados por estudos internacionais, como o PISA - Programa de Avaliação Internacional de Estudantes (Este programa visa avaliar a capacidade dos jovens de 15 anos no uso dos seus conhecimentos, de forma a enfrentarem os desafios da vida real, em vez de simplesmente avaliar o domínio que detêm sobre os conteúdos do seu currículo escolar específico. É uma avaliação via questionário, uma avaliação de cariz pedagógico), desenvolvido no âmbito da Organização para o Desenvolvimento e Cooperação Económico (OCDE, 1997) (http://www.gave.min-edu. pt/np3/157.html), não colocam as competências de leitura dos portugueses numa situação muito favorável, apesar dos progressos registados nos últimos anos (http://www.gave.min-edu.pt/np3/271.html).
Desta forma, procurando dar respostas aos baixos níveis de literacia em Portugal relativamente à média europeia, o Ministério da Educação, juntamente com o Ministério da Cultura, cria, em 2006, o Plano Nacional de Leitura, visando a aquisição, a promoção e a intervenção nas competências e hábitos de leitura junto da população juvenil (Roque, 2011).

Com base nestas decisões, tornou-se ainda mais indispensável a criação e/ou aferição de instrumentos adequados para a avaliação psicológica das competências de leitura dos alunos do ensino básico, de forma a colmatar as necessidades identificadas e estimular o progresso de cada aluno em particular (Sim-Sim \& Viana, 2007).

Face à "escassez de medidas nacionais fiáveis, válidas e aferidas nacionalmente" (Sim-Sim \& Viana, 2007), pretende-se com o presente trabalho contribuir para a aferição portuguesa (Figueira \& Lopes, n.d) de uma Bateria de Avaliação dos Processos de Leitura para Crianças, a PROLEC-R (Cuetos, Rodríguez, Ruano \& Arribas, 2009).

\section{MÉTODO}

\section{Objetivo}

O objetivo final do estudo é contribuir para a aferição portuguesa (Figueira \& Lopes, n.d) da Bateria de Avaliação dos Processos de Leitura para Crianças, a PROLEC-R (Cuetos, Rodríguez, Ruano \& Arribas, 2009), comparando o desempenho de crianças portuguesas e espanholas, do $5^{\circ}$ ano do ensino básico, de forma a conhecer as diferenças existentes no desempenho da leitura.

\section{Instrumento}

Bateria de Avaliação dos Processos de Leitura - Revista (Figueira \& Lopes, n.d., versão de investigação)

No que diz respeito ao instrumento, foi utilizada a versão de investigação (Figueira \& Lopes, n.d.) da PROLEC-R (Cuetos, Rodrígues, Ruano \& Arribas, 2009), versão revista da primeira versão de 1996.

Esta bateria é composta por nove provas que visam a avaliação do desempenho dos sujeitos na leitura, permitindo conhecer as suas capacidades e dificuldades. Todavia, é uma bateria que não avalia todos os processos considerados, hoje, importantes na avaliação cabal das capacidades leitoras, enfatizando as questões de compreensão. A avaliação é realizada por meio da análise das respostas a quatro processos: Reconhecimento de Letras, Processos Léxicos, Processos Sintáticos e Processos Semânticos. 
O processo Reconhecimento de Letras envolve a primeira e a segunda prova da bateria. Na primeira prova - Nome ou Som das Letras - a criança deve fazer a leitura de 23 letras do alfabeto, sendo que três destas servem apenas de exemplo. A leitura das restantes 20 letras é cronometrada. Pretende compreender se a criança conhece todas as letras apresentadas. A cotação desta prova situa-se no intervalo de 0 a 20 pontos, sendo 1 ponto por cada resposta correta (a precisão). A velocidade é um dos processos também avaliada nesta prova, sendo pelo tempo que o sujeito utiliza na sua realização.

A segunda prova - Igual-Diferente - é constituída por 20 pares de palavras e pseudopalavras, sendo metade destes pares iguais (constituídos por palavras e/ou pseudopalavras iguais) e metade diferentes (constituídos por palavras e/ou pseudopalavras diferentes). Igualmente cronometrada, esta prova pre-tende analisar a atenção do sujeito e a sua capacidade de segmentação e reconhecimento das palavras. A pontuação varia também de 0 a 20 pontos, correspondendo 1 ponto a cada acerto.

Os Processos Léxicos aglutinam a terceira e a quarta prova. A terceira prova - Leitura de Palavras - consiste na leitura cronometrada de 40 palavras, sendo 13 dessas palavras muito frequentes, 14 de frequência média e as restantes 13 de uso pouco frequente (a partir da Corlex_base de dados lexicais). A estrutura silábica das palavras utilizadas é bastante variável, ao apresentarem na primeira sílaba estruturas com complexidade distinta, segundo a combinação de vogais (V) e consoantes (C). Assim, as estruturas apresentadas são do tipo: $\mathrm{CV}, \mathrm{VC}, \mathrm{CVC}$, $\mathrm{CCV}, \mathrm{CVV}$. Os resultados da prova variam entre $0 \mathrm{e}$ 40 pontos, sendo cada resposta correta cotada com 1 ponto. O tempo de realização da prova também é tido em consideração, de forma a avaliar a precisão do sujeito.

A quarta prova - Leitura de Pseudopalavras consiste na leitura cronometrada de 40 pseudopalavras, ou seja, de palavras sem significado. É importante referir que as pseudopalavras apresentadas nesta prova são muito semelhantes às apresentadas na prova anterior (leitura de palavras), com a mesma extensão e grafia próxima. Os resultados também variam de 0 a 40 pontos. Esta prova tem como objetivo a avaliação da capacidade do sujeito em ler palavras sem significado. Permite, se assim o avaliador entender, analisar a capacidade do sujeito em juntar sílabas em palavras e igualmente a dicção e entoação.

A quinta e sexta prova representam os Processos Sintáticos. A quinta prova tem como finalidade avaliar a compreensão da criança na realização do processa- mento sintático de frases com diferentes estruturas gramaticais. É composta por 16 frases referentes a 4 estruturas gramaticais diferentes: Frase Ativa, Frase Passiva, Frase Relativa e Frase de Complemento Focalizado e Duplicado. Para cada frase são apresentadas 4 imagens ilustrativas, sendo que apenas uma corresponde à resposta correta, funcionando as demais como meio de distração. A cotação da prova consiste na soma dos acertos, podendo variar de 0 a 20 pontos.

A sexta prova - Sinais de Pontuação - consiste na leitura de um texto que integra 11 sinais de pontuação (4 pontos, 1 vírgula, 3 exclamações e 3 interrogações). Esta leitura pretende avaliar a leitura dos sinais de pontuação que se encontram no texto. De leitura igualmente cronometrada, a pontuação pode variar de 0 a 1 ponto, conforme a resposta incorreta ou correta. A cotação máxima será de 1 ponto.

Por último, os Processos Semânticos englobam a sétima, oitava e nona prova. A sétima prova - Compreensão de Frases - tem por objetivo a verificação das capacidades da criança em retirar significado das frases apresentadas (16 frases), nas quais deve realizar algumas tarefas. Desta forma, as três primeiras tarefas destinam-se a ordens simples do tipo "dá três pancadas na mesa". As tarefas seguintes requerem que a criança faça desenhos simples, como, por exemplo, "desenha uma árvore com três maçãs". As seguintes tarefas remetem para a execução de pequenos retoques nos desenhos apresentados como, por exemplo, "desenha um chapéu no palhaço". Nas restantes tarefas, a criança deve ler e identificar a imagem (de quatro) que representa o que foi lido. A pontuação obtida nesta prova pode variar de 0 a 16 pontos, correspondendo cada resposta certa a um ponto.

A oitava prova - Compreensão de Textos - é composta por quatro textos (dois narrativos e dois expositivos), lidos pelo sujeito e para os quais são feitas perguntas sobre os mesmos, de forma a analisar a compreensão de cada texto lido. Esta prova contém quatro perguntas para cada um dos textos lidos, variando de 0 a 16 pontos a sua cotação ( 1 ponto por cada acerto).

A nona e última prova - Compreensão Oral - é constituída por dois textos expositivos, lidos em voz alta pelo examinador, pretendendo avaliar a compreensão da criança relativamente ao que ouve. Para cada texto, são colocadas quatro questões sobre o texto lido pelo examinador, variando a pontuação de 0 a 8 pontos, um ponto para cada resposta correta.

As pontuações alcançadas por cada criança dividem-se em dois tipos de índices: os Índices Princi- 
pais - com as informações mais importantes sobre as competências de leitura da criança, e os Índices Secundários - com informações sobre a Precisão, a Velocidade de Leitura das crianças.

\section{Amostra}

A amostra desta investigação é composta por dois grupos: Um de 50 alunos portugueses com frequência no $5^{\mathrm{O}}$ ano do ensino básico de uma instituição privada do centro do país (Região Urbana), possuindo como língua materna o português de Portugal, todos tendo frequentado o jardim de infância, pelo menos um ano, e outro que corresponde aos 151 alunos do $5^{\mathrm{O}}$ ano do ensino básico da amostra espanhola, com frequência em instituições públicas e privadas, provenientes das diversas regiões do país (Cuetos, Rodríguez, Ruano, \& Arribas, 2009), tendo como $1^{\text {a }}$ língua o espanhol, de espanha, tendo frequentado, na sua maioria, o jardim escola. Relativamente ao primeiro grupo $(\mathrm{N}=50)$, é constituído por 27 alunos do sexo masculino e 23 do sexo feminino.

Relativamente à amostra espanhola, o manual da PROLEC-R (Cuetos, Rodríguez, Ruano, \& Arribas, 2009) refere que a bateria foi aplicada a 151 crianças do $5^{\circ}$ ano de ambos os sexos (73 do sexo masculino e 78 do sexo feminino).

\section{Procedimentos}

Para a realização do presente estudo, foi utilizada toda a bateria PROLEC-R (Figueira \& Lopes, n.d.).

Primeiramente, foi solicitada autorização junto da direção do colégio, que foi concedida no início de fevereiro de 2013, com a indicação de que nos deveríamos dirigir aos encarregados de educação e aos alunos, a fim de agendar a aplicação das provas. Seguidamente, foram enviados os pedidos de autorização aos encarregados de educação de todos os alunos da amostra. Após algum tempo de espera pela receção das autorizações dos encarregados de educação, foram iniciadas as aplicações.

A administração da bateria foi realizada individualmente. O tempo de aplicação das provas foi pouco variável, atingindo em média 45 minutos. É de salientar que este tempo foi inflacionado devido à curiosidade apresentada por parte de todas as crianças. Após a conclusão de todas as recolhas de dados, procedeu-se à construção da base de dados.

\section{APRESENTAÇÃO DOS RESULTADOS}

Comparando os resultados obtidos na PROLEC-R, entre a amostra portuguesa (PT) e a amostra espanhola (ES), verificamos (Tabela 1),

TABELA 1

Síntese dos resultados, nas diferentes provas, nos dois grupos

\begin{tabular}{ccccccc}
\hline & \multicolumn{5}{c}{ Portugueses } & \multicolumn{2}{c}{ Espanhóis } \\
\hline Provas & máximo & mínimo & $\begin{array}{c}\text { Precisão } \\
\text { (média de acertos) }\end{array}$ & $\begin{array}{c}\text { Velocidade } \\
\text { (segundos) }\end{array}$ & $\begin{array}{c}\text { Precisão } \\
\text { (média de acertos }\end{array}$ & $\begin{array}{c}\text { Velocidade } \\
\text { (segundos) }\end{array}$ \\
\hline NL & 20 & 0 & 19.82 & 14.38 & 19.67 & 15.31 \\
LP & 40 & 0 & 39.82 & 41.08 & 39.62 & 33.19 \\
LS & 40 & 0 & 38.26 & 62.70 & 37.93 & 56.17 \\
ID & 20 & 0 & 18.86 & 41.18 & 19.09 & 53.80 \\
SP & 11 & 0 & 10.78 & 39.52 & 10.32 & 43.17 \\
EG & 20 & 0 & 12.74 & & 14.16 & 15.79 \\
CF & 16 & 0 & 15.68 & & 12.80 & \\
CT & 16 & 0 & 13.86 & 5.19 & \\
CO & 8 & 0 & 5.74 & & & \\
\hline
\end{tabular}


Relativamente às provas Nome de Letras (NL) e Leitura de Palavras (LP), estas foram as provas com maiores números de acertos, em ambas as amostras, sendo que a portuguesa apresenta, em ambas, uma média um pouco mais elevada de acertos (Nome de Letras: PT, $M=19,82$; ES, $M=19,67$; Leitura de Palavras: PT, $M=39,82$; ES, $M=39,62$ ). Relativamente à velocidade de leitura utilizada pelos sujeitos na prova NL, as crianças portuguesas apresentaram uma leitura mais rápida $(\mathrm{M}=14,38)$, comparada com a amostra espanhola, com uma média de $\mathrm{M}=15,31$ segundos. Na prova LP, a amostra espanhola, apesar de ter um resultado de precisão inferior ao resultado alcançado pela amostra portuguesa, apresenta uma maior velocidade de leitura (PT: $\mathrm{M}=41,08$; ES: $M=33,19$ ).

A prova Leitura de Pseudopalavras (LS) é, seguidamente, a prova com maior número de acertos, estando a amostra espanhola uma vez mais $(\mathrm{M}=37,93)$ abaixo da amostra portuguesa $(38,26)$. Quanto ao índice de velocidade, a amostra espanhola volta a apresentar um menor tempo de leitura $(M=56,17)$ comparativamente à amostra portuguesa, que apresenta uma média de $\mathrm{M}=62,70$ segundos.

Em relação à prova Igual-Diferente (ID), a amostra espanhola apresenta alguma vantagem face à amostra portuguesa, obtendo uma média de precisão de $M=19,09$ e a portuguesa uma média de 18,86 . No que diz respeito à velocidade, são os alunos portugueses que leem mais rapidamente $(M=41,18)$, alcançando os alunos espanhóis uma média de velocidade de $\mathrm{M}=53,80$.

Na prova Sinais de Pontuação (SP), ambas as amostras apresentam, novamente, valores próximos, porém, as crianças portuguesas alcançam uma média de $M=10,78$ face a uma média de $M=10,32$, das crianças espanholas. A velocidade de leitura é superior na amostra portuguesa, alcançando uma média de $M=39,52$, face à amostra espanhola, com uma média de $M=43,17$.

As Estruturas Gramaticais (EG) é a prova com maior diferença de resultados, alcançando a amostra portuguesa uma média de $\mathrm{M}=12,74$ e a amostra espanhola uma média de $M=14,16$, destacando-se, assim, com um maior número de acertos.

A prova Compreensão de Frases (CF), mais uma vez, apresenta valores proximais entre as duas amostras, obtendo a amostra portuguesa, novamente, uma média um pouco inferior (PT: $M=15,68$; $E S: M=15,79$ ).

Nas duas restantes provas, Compreensão de Textos (CT) e Compreensão Oral (CO), apesar dos resultados se mostrarem baixos, a amostra portuguesa, na primeira prova $(\mathrm{CT})$, apresenta uma média de
$M=13,86$ face a uma média de $M=12,80$, na amostra espanhola; e, na segunda prova (CO), uma média de $\mathrm{M}=5,74$, apresentando a amostra espanhola uma média de $\mathrm{M}=5,19$.

Fazendo uma comparação entre sexos, apenas para a amostra portuguesa, visto o manual espanhol da PROLEC-R (Cuetos et al., 2009) não apresentar dados comparativos, podemos observar que na prova Nome ou Som de Letras, os sujeitos do sexo feminino, apesar de apresentarem uma leitura mais rápida $(\mathrm{F}: \mathrm{M}=14$, 17 / $\mathrm{M}: \mathrm{M}=14,56)$, alcançam um menor número de acertos (F: $M=19,78 / M: M=19,85)$.

Na prova Igual-Diferente (ID), são os sujeitos do sexo feminino a apresentar uma leitura mais precisa ( $F: M=19,04$ / $M: M=18,70)$, porém, ligeiramente mais lenta, comparativamente aos sujeitos do sexo masculino ( $\mathrm{F}: \mathrm{M}=41,74$ / $\mathrm{M}: \mathrm{M}=40,70$ ).

A prova Leitura de Palavras (LP) apresenta um maior número de acertos para as meninas ( $F: M=39,91$ / $\mathrm{M}: \mathrm{M}=39,74)$ e uma maior rapidez para os meninos (F: $\mathrm{M}=41,78 / \mathrm{M}: \mathrm{M}=40,48)$.

Os resultados alcançados na prova Leitura de Pseudopalavras (PS) indicam que os sujeitos femininos leem de forma mais rápida $(\mathrm{F}: \mathrm{M}=62,04 / \mathrm{M}$ : $M=63,26$ ), porém, são os sujeitos do sexo masculino que leem de forma mais precisa, alcançando um nível de precisão de $\mathrm{M}=38,85$ face a uma média de $\mathrm{M}=37,57$ feminina.

Na prova Estruturas Gramaticais (EG), os sujeitos masculinos voltam a obter um maior número de acertos $(\mathrm{M}=12,85)$, comparativamente aos sujeitos femininos $(\mathrm{M}=12,61)$.

Na prova Sinais de Pontuação (SP), encontramos resultados interessantes, pois tanto os sujeitos do sexo feminino, como os sujeitos do sexo masculino, conseguiram os mesmos resultados de precisão $(\mathrm{M}=10,78)$, diferenciando apenas no que diz respeito à velocidade de leitura ( $\mathrm{F}: \mathrm{M}=38,70 / \mathrm{M}: \mathrm{M}=40,22)$.

Nas últimas três provas (Compreensão de Frases, Compreensão de Textos e Compreensão Oral), são, mais uma vez, os sujeitos do sexo masculino a alcançar resultados mais precisos.

\section{DISCUSSÃO E CONCLUSÕES}

Assume-se que a importância da leitura é indiscutível. Através dela podemos obter sucesso escolar, profissional e social (Borges, 1998; Carvalho \& Pereira, 2008; Sousa, 1999). No entanto, a leitura não é adquirida de forma espontânea (Silva, 2003). É uma atividade complexa que resulta dos diversos processos cognitivos que o leitor utiliza perante a construção do sentido de um texto (Campos, 2008; Cruz, 2007). 
Face à escassez de medidas nacionais fiáveis, válidas e aferidas nacionalmente (Sim-Sim \& Viana, 2007), o objetivo deste estudo prendeu-se, fundamentalmente, com a aferição portuguesa da PROLEC-R, instrumento que permitirá avaliar, de forma válida e fiável, a partir dos dados normativos, alguns dos processos cognitivos utilizados pelo indivíduo na leitura, como também fornecer as medidas necessárias para uma intervenção adequada à necessidade específica de cada um, estimulando o progresso de cada aluno em particular. Para além do contributo para a aferição portuguesa, com a recolha de dados junto de crianças do $5^{\circ}$ ano de escolaridade, realizámos uma comparação qualitativa entre as crianças portuguesas e as crianças espanholas, do 5으o ano do ensino básico, de forma a conhecer as potenciais diferenças existentes no desempenho de leitura.

Pela observação dos resultados, registamos quais as dificuldades/competências dos alunos portugueses relativamente à bateria de avaliação dos processos cognitivos implicados na leitura. Podemos verificar uma acentuada dificuldade dos alunos de ambas as amostras nas provas Estruturas Gramaticais (EG), Compreensão de Textos (CT) e Compreensão Oral, sendo que estas provas não foram respondidas corretamente pela maioria dos sujeitos.

Os resultados obtidos poderão refletir, no entanto, vários aspetos: o entendimento inapropriado das palavras utilizadas nas frases/textos apresentados (exemplos de palavras pouco habituais assinaladas pelos sujeitos: "repreende-a", "saúda-o", "acaricia-o", "consolála", "pradaria", "encurralando-os", "desfiladeiros", "precipícios", "adotaram", "expedições"); a falta de conhecimentos prévios sobre os domínios neles tratados (nunca ouviram falar de "okapis", sobre os "índios Apaches" ou sobre o "ratel"); a ausência de familiaridade por parte dos alunos com a estrutura sintática ("O gato, ataca-o o rato", ao invés de "O rato ataca o gato", estrutura mais utilizada nas escolas) e uma deficiência dos recursos prosódicos (nota-se que na prova Sinais de Pontuação é pedido à criança que leia com "clareza e com boa entoação, como se estivesses a contá-lo aos teus amigos", o que não acontece na prova Compreensão de Textos).

Outro aspeto diz respeito à concentração e atenção que as crianças disponibilizaram às provas. Por ser a PROLEC-R uma bateria extensa, que exige da criança avaliada uma grande capacidade de concentração e reflexão e visto a aplicação das provas terem ocorrido após o almoço, durante o recreio ou mesmo no fim do tempo letivo, acreditamos que alguns resultados refletem o desejo das crianças em "despachar" tudo o mais rápido possível, a fim de poderem ter tempo para brincar ou simplesmente irem para casa. Assim, seria recomendável que a aplicação da bateria fosse realizada pela manhã, de forma a manter a atenção e a concentração das crianças nas provas aplicadas.

Um outro aspeto prende-se com a ordem das provas apresentadas aos alunos, o que parece influenciar os resultados obtidos, visto as provas em que apresentam mais dificuldades serem as três provas de grande complexidade, e as últimas. A sugestão que propomos (com base no resultados obtidos) implicaria uma aplicação com níveis de complexidade distribuídos alternadamente, por exemplo: 1. Prova Nome ou Som de letras (NL); 2. Prova Estruturas Gramaticais (EG); 3. Prova Leitura de Palavras (LP); 4. Prova de Compreensão Oral (CO); 5. Prova Compreensão de Frases (CF); 6. Prova Leitura de Pseudopalavras (LS); 7. Prova Sinais de Pontuação (SP); 8. Prova IgualDiferente (ID); e, 9. Prova Compreensão de Textos (CT).

Visto este estudo ser exploratório, estudos adicionais deverão ser realizados para validar e corroborar os presentes resultados obtidos. Além disso, é necessário ter em atenção que a amostra portuguesa do presente estudo é constituída apenas por sujeitos de meio urbano, com frequência numa instituição de caráter privado, contrariamente à amostra espanhola, que abrange sujeitos de diferentes regiões e de ensino público e privado. Desta forma, é importante que futuros estudos sejam realizados noutros contextos, nomeadamente com sujeitos de meios rurais e de ensino público e com uma amostra maior de sujeitos, por forma a refutar os resultados.

O objetivo deste estudo centrou-se, essencialmente, no contributo para a aferição portuguesa da PROLEC-R, fazendo-se uma avaliação dos processos cognitivos implicados na leitura, comparando qualitativamente os resultados obtidos por ambas as amostras a fim de verificar a existência de diferenças no processo de desempenho de leitura.

Ao contrário de outros estudos já realizados (eg. PISA, que, por razões óbvias, não conseguimos apresentar neste artigo), em que os alunos espanhóis obtêm resultados mais positivos, nesta investigação, os alunos da amostra portuguesa alcançaram resultados superiores comparativamente aos alunos da amostra espanhola, com a PROLEC-R.

É importante referir que a amostra portuguesa tem frequência numa instituição privada, da região centro do País, sendo todos os alunos integrantes da amostra de meio urbano. Neste sentido, e sendo a amostra espanhola proveniente das diversas regiões do país, de ensino público e privado, torna-se fundamental avaliar o desempenho de leitura dos alunos das diferentes 
regiões e dos diferentes ensinos, a fim de conseguir uma comparação mais equitativa.

Quanto às limitações do presente estudo, estas prenderam-se com a indisponibilidade dos alunos na resolução da bateria, o extenso tempo de aplicação, visto estes terem um horário escolar muito preenchido e rígido e a falta de permissão dos encarregados de educação, acreditando muitos pais que estas avaliações pudessem ser constrangedoras ou mesmo taxativas.

\section{REFERÊNCIAS}

Borges, A. G. S., Assagra, A. G., \& Alda, C. G. L. (2010). Leitura: o mundo além das palavras. Curitiba: Instituto RPC. Campos, G. P. C. (2008). O processo de leitura: da descodificação à interação. Revista Objetiva, 4, 56-65.

Carvalho, A. C. \& Pereira, M. M. (2008). Teste de Avaliação da Fluência e Precisão da Leitura: o Rei. Actas do $7^{0}$ Encontro Nacional (5ํㅡㄴ Internacional) de Investigação em Leitura, Literatura Infantil e Ilustração (pp. 155-169). Braga: Universidade do Minho.

Cruz, V. (2007). Uma abordagem cognitiva da leitura. Lisboa: Lidel.

Cuetos, F., Rodríguez, B., Ruano E., \& Arribas, D. (2009). Bateria de evaluacion de los processos lectores, revisada. Madrid: TEA Ediciones.

Esteves, M. (2009). Construção e desenvolvimento das competências profissionais dos professores. Sísifo. Revista de Ciências da Educação, 8, 37-48.

Figueira, A. P. \& Lopes, I. P. (n.d). Bateria de Avaliação dos Processos de Leitura para Crianças, a PROLEC-R (versão de investigação, CEGOC).

Leite, I. \& Bragança, A. (2010). Estudo dos manuais escolares da língua portuguesa para o $1^{\mathrm{O}}$ ano de escolaridade. In R. Beard, L. S. Siegel, I. Leite, \& A. Bragança (Ed.). Como se aprende a ler? (cap. 4, pp. 99-140). Lisboa: Fundação Francisco Manuel dos Santos.

Merçon, J. (2013). Desempenho na leitura (a partir da PROLEC-R): estudo comparativo entre crianças portuguesas e espanholas. Análise exploratória. Dissertação de Mestrado, não publicada, apresentada à Faculdade de Psicologia e de Ciências da Educação da Universidade de Coimbra.

Paulino, J. I. B. (2009). Consciência fonológica: implicações na aprendizagem da leitura. Dissertação de Mestrado apresentada à Faculdade de Psicologia e Ciências da Educação da Universidade de Coimbra.

Roque, E. (2011). A PROLEC-R (Bateria de Avaliação dos Processos de Leitura para Crianças). Contributo para a sua adaptação para a população portuguesa. Estudo exploratório com crianças do 5o ano de escolaridade. Dissertação de Mestrado, não publicada, apresentada à Faculdade de Psicologia e de Ciências da Educação da Universidade de Coimbra.

Silva, A. C. (2003). Até a descoberta do princípio alfabético. Lisboa: Fundação Calouste Gulbenkian.

Sim-Sim, I. \& Viana, F. L. (2007). Para a avaliação do desempenho da leitura. Lisboa: Gabinete de Estatística e Planeamento da Educação.

Soares, M. B. (1991). Alfabetização no Brasil - o estudo do conhecimento. Brasília: Inep/Reduc.

Soares, M. (2000). As condições sociais da leitura: uma reflexão em contraponto. In R. Zilberman \& E. T. Silva (Org.). Leitura: perspectivas disciplinares (pp. 18-299). São Paulo: Ática.

Velásquez, M. G. F. (2002). Consciência fonológica e conceitos acerca da escrita: um estudo com alunos do $1^{\circ}$ ano de escolaridade. Dissertação de Mestrado apresentada ao Instituto de Educação e Psicologia da Universidade do Minho, Braga.

\footnotetext{
Autores:

Endereço para correspondência:

Ana Paula Couceiro Figueira

Universidade de Coimbra

Faculdade de Psicologia e de Ciências da Educação

Rua do Colégio Novo, 3030-301

Coimbra, Portugal

Recebido em: 09.06.14

Aceito em: 25.11 .14
}

Ana Paula Couceiro Figueira - Doutora, Faculdade de Psicologia e de Ciências da Educação da Universidade de Coimbra. JenNifer MERÇON - Mestre, Faculdade de Psicologia e de Ciências da Educação da Universidade de Coimbra. 